\title{
MODELLING THE GROWTH OF ZINC OXIDE NANOSTRUCTURES
}

\author{
JADE R. MACKAY ${ }^{\circledR}$, STEPHEN P. WHITE ${ }^{2}$ and SHAUN C. HENDY ${ }^{1,2}$
}

(Received 5 November, 2007; revised 29 January, 2008)

\begin{abstract}
Zinc oxide is known to produce a wide variety of nanostructures that show promise for a number of applications. The use of electrochemical deposition techniques for growing $\mathrm{ZnO}$ nanostructures can allow tight control of the morphology of $\mathrm{ZnO}$ through the wide range of deposition parameters available. Here we model the growth of the rods under typical electrochemical conditions, using the Nernst-Planck equations in two dimensions to predict the growth rate and morphology of the nanostructures as a function of time. Generally good quantitative and qualitative agreement is found between the model predictions and recent experimental results.
\end{abstract}

2000 Mathematics subject classification: primary 65M06.

Keywords and phrases: crystal growth, electrochemistry, zinc oxide, ZnO, NernstPlanck, simulation, model, finite difference.

\section{Introduction}

Zinc oxide is a material known to form a wide variety of nanostructures that show promise for applications in optoelectronics [10], sensors [1] and piezoelectronics [9]. As aqueous electrochemical deposition techniques often allow precise control of the morphology of oxide nanostructures, there is interest in applying these methods to $\mathrm{ZnO}[6]$. Indeed there is evidence that electrochemical parameters such as the substrate lattice parameter [13], the electrolyte [3, 4, 8], the amount of oxygen dissolved in the electrolyte [11], the applied potential across the cell [14], and electrode pretreatment [3] can all influence the morphology of electrochemically deposited $\mathrm{ZnO}$ [6]. Electrochemical synthesis is also attractive because it has the scope for large-scale industrial application [15] while being low-cost and readily accessible to researchers.

\footnotetext{
${ }^{1}$ MacDiarmid Institute, School of Chemical and Physical Sciences, Victoria University, Wellington, New Zealand; e-mail: jademackay@gmail.com.

${ }^{2}$ Industrial Research Limited, Lower Hutt, New Zealand; e-mail: s.hendy@irl.cri.nz, shaun.hendy@vuw.ac.nz.

(C) Australian Mathematical Society 2009, Serial-fee code 1446-1811/2009\$16.00
} 


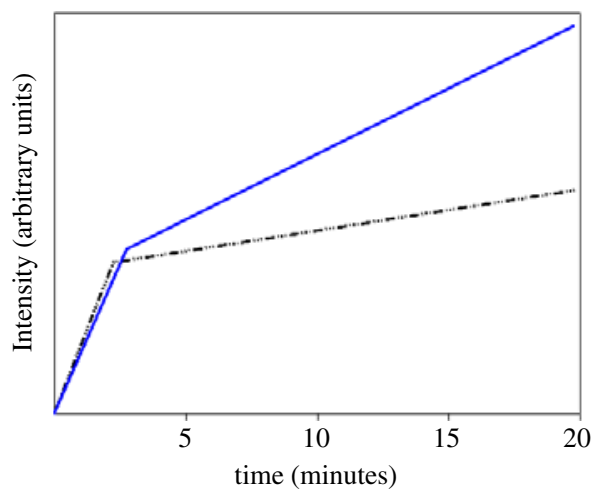

(a) High (solid) and low (dashed) cathodic potential.

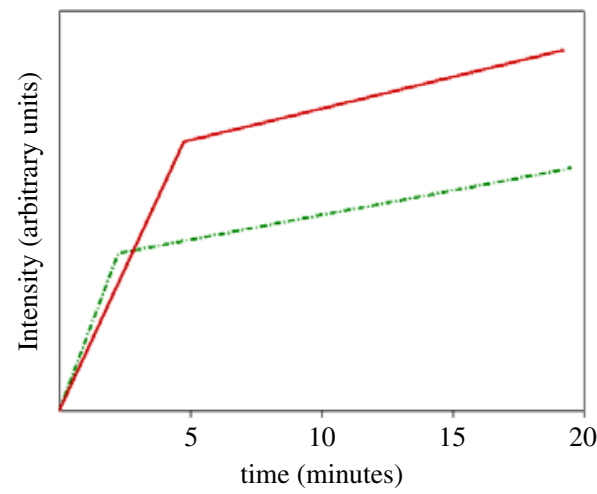

(b) High (dashed) and low (solid) $\mathrm{Zn}^{2+}$ concentration.

FIGURE 1. Schematic representations of the results obtained from x-ray absorption measurements of ZnO crystallite film growth with variation of (a) cathodic potential and (b) $\mathrm{Zn}^{2+}$ concentration.

In [6] $\mathrm{ZnO}$ nanostructured films were deposited by applying a cathodic potential bias to an electrode substrate immersed in an electrolyte at $80^{\circ} \mathrm{C}$. The electrolytic solution was composed of a small amount $(5 \mathrm{mM})$ of zinc chloride $\left(\mathrm{ZnCl}_{2}\right), 100 \mathrm{mM}$ calcium chloride $\left(\mathrm{CaCl}_{2}\right)$, and dissolved molecular oxygen. Typically, the resulting films consisted of an array of columnar hexagonal prismatic crystallites ("rods") each with a radius between 80 and $200 \mathrm{~nm}$ and length greater than $1000 \mathrm{~nm}$, depending on the deposition conditions. The crystallites were identified as having the $\mathrm{P}_{3} \mathrm{mc}$ crystal structure [18], a hexagonal structure often referred to as "wurtzite".

Subsequently, synchrotron radiation was used to measure the rate of $\mathrm{ZnO}$ deposition in situ under various electrochemical conditions [7]. A schematic representation of the observed growth rates is shown in Figure 1. These results show a clear difference between the effect of varying the potential and that of varying concentration of zinc ions. Both results show a short initial period (less than five minutes) of rapid growth followed by a long period of slower growth. At a given zinc concentration an increase in the magnitude of the cathodic potential resulted in no discernible difference in the rapid growth curve segment, but yielded a dramatic increase in the slope of the second phase curve segment. Increasing the zinc concentration at a given cathodic potential resulted in a shortening of the duration of the initial phase but little change in the initial growth rate, and only marginal differences between the growth rates of the second phase.

Here our goal is to model the growth of the rods under conditions as close as possible to the experiments described in [7] in terms of mass transport, using the Nernst-Planck equations and electroneutrality, to predict the growth rate and morphology of the nanostructures as a function of time. The information produced is then used to interpret the observed growth dependence on the growth conditions and the identification of critical parameters that control film morphology. 


\section{Electrochemistry}

In this section we give a brief summary of the basic electrochemical processes necessary to model the system. Dissolved zinc ions $\left(\mathrm{Zn}^{2+}\right)$ react with hydroxide $\left(\mathrm{OH}^{-}\right)$that is generated at the electrode by reduction of oxygen $\left(\mathrm{O}_{2}\right)$ with water $\left(\mathrm{H}_{2} \mathrm{O}\right)$ to form the insoluble metal oxide $\mathrm{ZnO}$. The formation of hydroxide occurs via the oxygen reduction reaction:

$$
\mathrm{O}_{2}+2 \mathrm{H}_{2} \mathrm{O}+4 \mathrm{e}^{-} \rightarrow 4 \mathrm{OH}^{-} \quad E=0.4 \mathrm{~V} / \mathrm{NHE}
$$

which leads to the formation of $\mathrm{ZnO}$ by:

$$
\mathrm{Zn}^{2+}+2 \mathrm{OH}^{-} \rightleftharpoons \mathrm{ZnO}+\mathrm{H}_{2} \mathrm{O} .
$$

Conditions that result in $\mathrm{Zn}$ metal formation

$$
\mathrm{Zn}^{2+}+2 \mathrm{e}^{-} \rightarrow \mathrm{Zn} \quad E=-0.76 \mathrm{~V} / \mathrm{NHE}
$$

must be avoided. Thermodynamic considerations allow the determination of an applied potential domain consistent with the above requirements. The potential boundaries are found to be $-0.76<E_{c}<+0.4 \mathrm{~V} / \mathrm{NHE}$ where $E_{c}$ is the applied cathodic potential and NHE denotes reference to the normal hydrogen electrode. We evaluate Reaction (2.2) on the crystal surfaces, and Reaction (2.1) at both the electrode and the crystal surfaces.

\section{Model}

The transport of $M$ aqueous charged species in an electric field under the constraints of electroneutrality $\left(\sum_{i}^{M} z_{i} c_{i}=0\right.$, where $c_{i}$ is the concentration and $z_{i}$ is the valence of species $i$ ) yields the following nonlinear system of partial differential equations [17]:

$$
\begin{gathered}
\frac{\partial c_{i}}{\partial t}=z_{i} u_{i} F \nabla \cdot\left(c_{i} \nabla \phi\right)+D_{i} \nabla^{2} c_{i} \\
\nabla \cdot(\sigma \nabla \phi)=F \sum_{i}^{M} z_{i} D_{i} \nabla^{2} c_{i} \quad \text { where } \sigma=F^{2} \sum_{i}^{M} z_{i}^{2} u_{i} \nabla c_{i} .
\end{gathered}
$$

Here $\phi$ is the electric potential, $F$ is Faraday's constant, while $u_{i}$ and $D_{i}$ are the mobility and diffusion coefficient of species $i$, respectively. The $M$ equations (3.1) are known as the Nernst-Planck equations. Implicit in this formulation is the electroneutrality condition. Frequently, one would use the electroneutrality condition to eliminate one species from the problem, and solve only the remaining $M-1$ Nernst-Planck equations. However, because the $\mathrm{CaCl}_{2}$ buffer, which is not involved in any of the electrode reactions, dominates the ionic strength of the electrolyte, we simply solve the three Nernst-Planck equations for the three species involved in the electrode reactions $\left(\mathrm{Zn}^{2+}, \mathrm{O}_{2}\right.$ and $\left.\mathrm{OH}^{-}\right)$. 


\section{BULK}

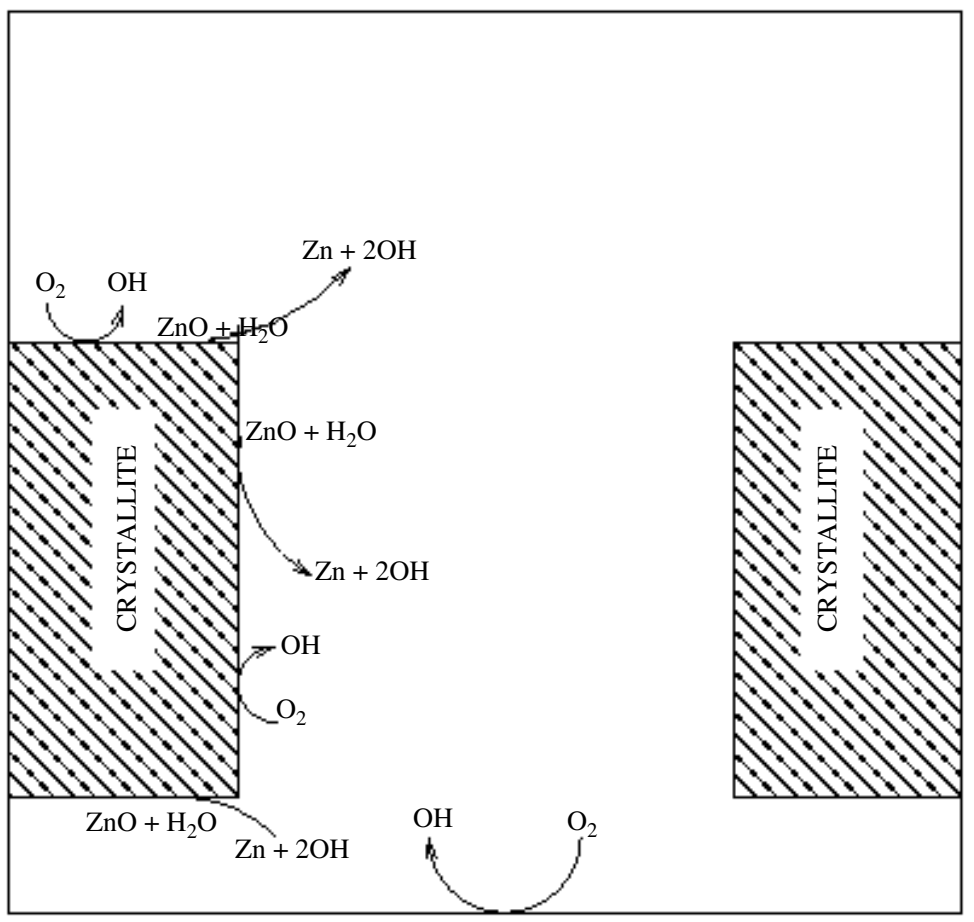

Electrode

FIGURE 2. Schematic of computational domain (white) and the relevant boundaries. Also shown are the reactions considered at various boundaries.

To simplify the problem we have solved this system numerically on a twodimensional domain with periodic boundary conditions in the direction perpendicular to the electrode as shown in Figure 2. This amounts to considering the growth of an array of infinitely long ridges rather than rods. In this study we have chosen to include only the full chemical reactions necessary for $\mathrm{ZnO}$ deposition, omitting intermediate reactions. Furthermore, we have constrained them to occur only at the appropriate boundaries, not in the electrolyte. In addition, periodic boundary conditions are applied at the domain sides to simulate an array of growing nanostructures, and bulk electrolyte concentrations are imposed at the top of the computational domain.

The precise mechanistic details of how the $\mathrm{ZnO}$ forms and the solution composition $\left(\mathrm{Zn}_{x} \mathrm{O}_{y} \mathrm{H}_{z}\right.$ complexes are known to exist) are still only known for a few specific conditions [16]. The model transport-reaction scheme is shown in Figure 3. The scheme depicts fluxes with vertical arrows and electrochemical reactions with horizontal arrows, and summarizes the paths that the bulk species must take for $\mathrm{ZnO}$ to be formed.

The details of both the chemical reactions (2.1) and (2.2) can be quite complex. Firstly, the oxygen reduction reaction is an electrode reaction, and therefore is 


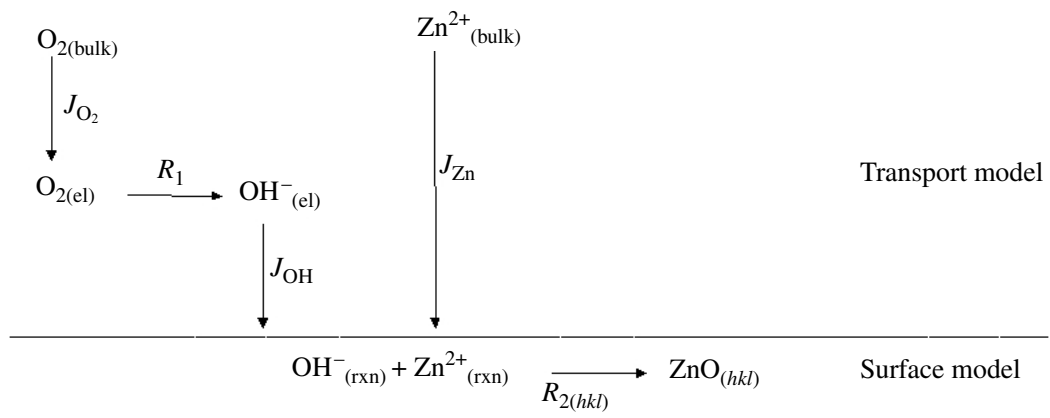

FIGURE 3. The constrained transport-reaction scheme for the species involved in $\mathrm{ZnO}$ formation with $\mathrm{Zn}^{2+} / \mathrm{OH}^{-}$reaction fixed at the electrode. Again (bulk) refers to the solution composition at great distance from the growing film, (el) is the electrode region, (rxn) is the region where $\mathrm{Zn}^{2+}$ and $\mathrm{OH}^{-}$meet, and $(h k l)$ refers to a particular $\mathrm{ZnO}$ crystal facet. Transport is denoted with vertical arrows and chemical reactions with horizontal arrows.

influenced by the electrode overpotential. The accepted treatment is to use the ButlerVolmer equation under the assumption of low field conditions [19]. However, in the $\mathrm{ZnO}$ system at the potentials we are investigating, the oxygen reduction reaction proceeds very close to the diffusion limited regime [5]. Therefore, in this study we assume the $\left[\mathrm{O}_{2}\right]_{\text {electrode }}=0$ as the electrode boundary condition for $\mathrm{O}_{2}$.

The reaction and incorporation of $\mathrm{ZnO}$ units into the growing crystal is also a complex process which involves the surface charge of the growing crystal, the electric double layer, the dynamics of the crystal surface features (such as steps and kinks), and the multitude of intermediate steps between adsorption and inclusion. In this study we use a simple scaling rate law of the type

$$
R_{h k l}=\alpha_{h k l}\left(1-\frac{Q}{K}\right),
$$

where $R_{h k l}$ is the rate of inclusion of $\mathrm{ZnO}$ units in $\mathrm{mol} \mathrm{s}^{-1}, \alpha_{h k l}$ is the surface specific reaction-inclusion rate constant, and $Q$ is the reaction quotient corresponding to the reverse of Reaction (2.2),

$$
Q=\left[\mathrm{Zn}^{2+}\right]\left[\mathrm{OH}^{-}\right]^{2},
$$

where square brackets denote concentrations. The magnitude of the rate coefficients $\alpha_{h k l}$ can be estimated to match the experimental growth rates in [7]. Note that the forward form

$$
Q=\frac{1}{\left[\mathrm{Zn}^{2+}\right]\left[\mathrm{OH}^{-}\right]^{2}}
$$

is problematic if any concentration is zero. Finally, $K$ is the equilibrium (dissolution) constant. It is easy to see that when $Q>K \Rightarrow R<0$ the rate of production of $\mathrm{Zn}^{2+}$ and $\mathrm{OH}^{-}$is negative, that is, $\mathrm{ZnO}$ is produced. Conversely when $Q<K \Rightarrow R>0$ the rate of production of $\mathrm{Zn}^{2+}$ and $\mathrm{OH}^{-}$is positive and $\mathrm{ZnO}$ dissolution is favoured. 
In the wurtzite structure, $\mathrm{ZnO}$ predominantly exhibits two families of crystal facets: the $\{0001\}$ (which is polar) and the $\{01 \overline{1} 0\}$ (which is nonpolar). The anisotropy of the different crystal surfaces is modelled here by assigning different values of $\alpha_{h k l}$ for each facet. Furthermore, changes in the applied potential will also alter the reaction rates at the different crystal facets, an effect we mimic in this paper by varying the rate constants $\alpha_{h k l}$. The boundary conditions for the ionic flux of species $i$ at the $(h k l)$ crystal facet are then given by

$$
A_{h k l} \vec{n}_{h k l} \cdot\left(\vec{J}_{i}+c_{i} \vec{v}_{h k l}\right)=R_{h k l}
$$

where $\vec{n}_{h k l}$ is the unit normal to the facet, $v_{h k l}$ is the velocity of the facet, $R_{h k l}$ is the rate of $\mathrm{ZnO}$ formation and $A_{h k l}$ is the facet area.

The model parameters $D_{i}$ and $K$ can be found in the literature. We have calculated $K$ to be $10^{-7.65}$ from the Gibbs free energy of formation [2]. The diffusion coefficients of $\mathrm{Zn}^{2+}\left(1.4 \times 10^{-5} \mathrm{~cm}^{2} \mathrm{~s}^{-1}\right)$ and $\mathrm{OH}^{-}\left(5.3 \times 10^{-5} \mathrm{~cm}^{2} \mathrm{~s}^{-1}\right)$ were obtained from the reference literature [12] and $D_{\mathrm{O}_{2}}\left(3.4 \times 10^{-5} \mathrm{~cm}^{2} \mathrm{~s}^{-1}\right)$ was obtained from the electrochemical literature [5]. The magnitude of the $\alpha_{h k l}$ parameters was chosen so that the simulations grew at approximately the rates observed in experiments; nonetheless, these parameters were varied in order to understand their effect on the growth regimes.

\section{Numerical method}

We used the method of finite differences with a rectangular mesh to solve the system of equations for the three reactive species. Central differences were used for all differential operators, giving second-order spatial accuracy on the uniform mesh. To reduce computational overhead we used a nonuniform mesh away from the growing crystal (shown in Figure 4). The mesh rules used were

$$
\begin{cases} \begin{cases}x_{m}=(m-1) \Delta x, & m<m_{c}, \\ x_{m}=x_{m-1}+2 a_{m-1} \Delta x, & m>m_{c},\end{cases} \\ \begin{cases}y_{n}=(n-1) \Delta y, & n<n_{c}, \\ y_{n}=y_{n-1}+2 a_{n-1} \Delta y, & n>n_{c},\end{cases} \end{cases}
$$

where $\Delta x$ and $\Delta y$ are the $x$ and $y$ fine grid increments, $m_{c}$ and $n_{c}$ are the indices after which the grid becomes nonuniform and $a_{n-1}=\sum_{k=0}^{n-2} a_{k}$ and $a_{m-1}=\sum_{k=0}^{m-2} a_{k}$ are responsible for nonuniformity. In the subsequent calculations, critical indices $m_{c}$ and $n_{c}$ were set to 5 which corresponds to $25 \mathrm{~nm}$ of fine mesh surrounding the crystallite. The mesh was updated when the amount of material having crossed the boundary exceeded the amount of material encased in one fine mesh increment. The new solution vector is acquired by linear interpolation (weighted averaging) of the four bounding points from the previous mesh. 


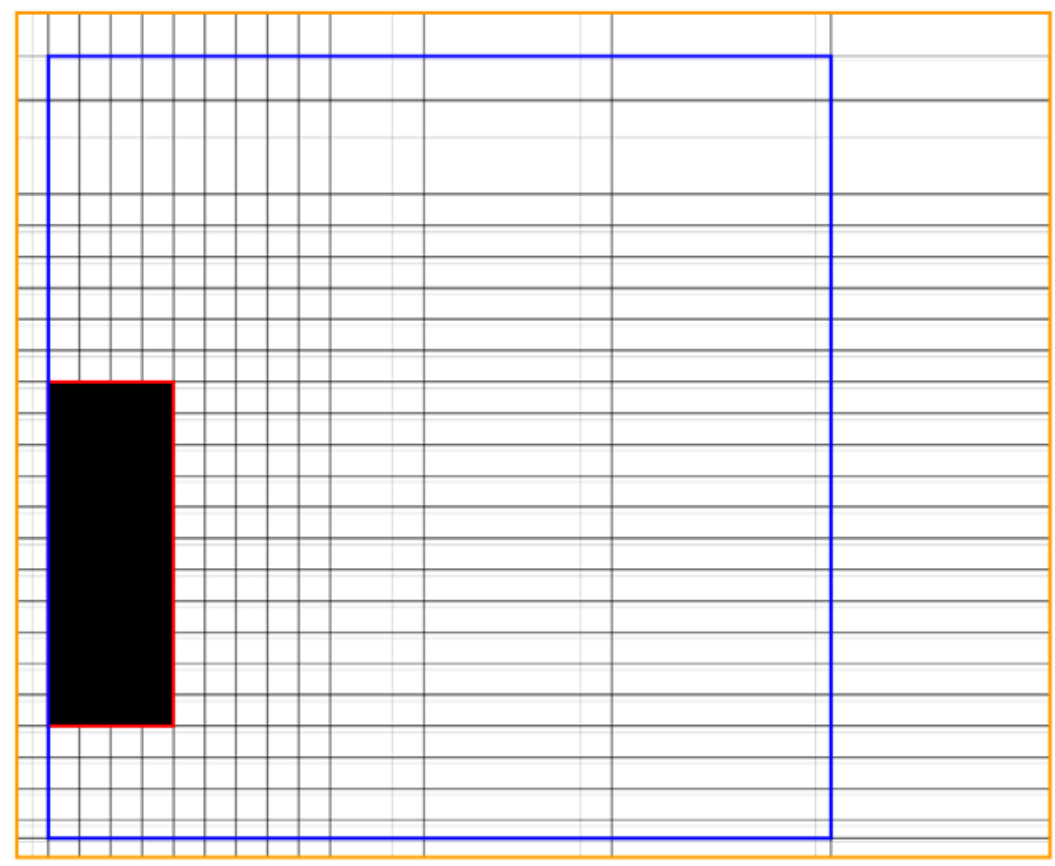

FIGURE 4. Illustration of a nonuniform mesh. Here the outer boundary corresponds to the artificial $x+1$ and $y+1$ points used to evaluate Neumann-like boundary conditions, the dark rectangle inside the boundary is the NP domain boundary and the filled rectangle represents the crystal. The dark gridlines are the present grid, and the fainter grey lines indicate the grid belonging to the previous interpolant.

Dirichlet conditions were implemented indirectly as the central average of adjacent neighbours, resulting in a boundary condition with very similar structure to a Neumann boundary condition. For example, the Dirichlet condition

$$
c_{0}=p
$$

was approximated as the central average of its neighbours

$$
c_{0} \approx \frac{c_{-1}+c_{+1}}{2}
$$

where $c_{-1}$ is a ghost point outside the physical domain. The boundary condition $c_{0}=p$ is now evaluated at $c_{-1}$ by rearranging $\left(c_{-1}+c_{+1}\right) / 2=p$ to $c_{-1}=2 p-c_{+1}$, allowing all boundary conditions to be coded with the same structure. This method also ensures that the boundary condition is evaluated at same the point for both Neumann and Dirichlet boundary conditions. Comparison of results obtained from the direct and indirect Dirichlet implementations found the solutions to be identical, and a marginal improvement in convergence for the indirect method.

To move forward in time we used Newton's algorithm to iteratively solve the implicit time discretized difference equations. We used the Cranck-Nicolson time discretization for the Nernst-Planck equation (3.1) and the fully implicit limit for the 
charge continuity equation (3.2). The resulting system of linear equations was solved using an LU-decomposition (implemented with the LAPACK dgesv routine). The solution was then used as the guess for the next time step. We used a time increment of $1 \mathrm{~s}$, and a grid increment of $5 \mathrm{~nm}$ for the uniform mesh region. The spatial domain has real space dimensions of height between 500 and $1000 \mathrm{~nm}$ and width between 40 and $100 \mathrm{~nm}$. A typical mesh consists of around 1600 points. However, as the crystal grows into the domain this number is reduced. We observe the desired quadratic convergence of Newton's method and, using an appropriate error criterion, we typically obtained convergence within two to three iterations.

\section{Results}

Our goal is to relate the individual facet growth behaviour to the experimentally observed growth profiles. The real time duration of our simulations is approximately 20 minutes or less, with the simulations being stopped when the neighbouring crystallites meet. Our first simulations sought to locate the magnitude of the facet rate constants observed experimentally. We found that use of the mass transport limited extreme for $\mathrm{ZnO}$ formation at the lateral facet,

$$
\left[\mathrm{Zn}^{2+}\right]_{\text {side }}=0,
$$

resulted in a phase one growth curve very similar to the experimental measurements. The result demonstrates the important role of mass transport in determining the outcome of the crystallization. Since the mass transport limited lateral growth yields a good match to the phase one growth it provides a good basis for further investigation of the second growth phase.

Further simulations were undertaken investigating the effect of varying the rate of $\mathrm{ZnO}$ formation on the top crystal surface whilst keeping the lateral growth rate fixed at the mass transport limited rate. Applying the mass transport limited growth condition to the lateral boundary gives us a phase one growth curve consistent with experiment. Figure 5 shows the growth profiles obtained using $\alpha_{\text {top }}=1 \times 10^{-8} \mathrm{~mol} \mathrm{~s}^{-1}, \alpha_{\text {top }}=$ $2 \times 10^{-8} \mathrm{~mol} \mathrm{~s}^{-1}, \alpha_{\text {top }}=5 \times 10^{-8} \mathrm{~mol} \mathrm{~s}^{-1}, \alpha_{\text {top }}=1 \times 10^{-7} \mathrm{~mol} \mathrm{~s}^{-1}$, and the mass transport limited $\mathrm{ZnO}$ formation condition $\left(\left[\mathrm{Zn}^{2+}\right]_{\text {face }}=0\right)$. Faster vertical growth is observed for greater top-face reaction rates. The lateral growth contribution to phase two growth is greater for the slower top-face rates, as we might expect due to the greater abundance of reactants associated with slower consumption at the top face. However, growth at the top dominates during phase two growth, so that the reduced lateral growth only slightly reduces the volumetric growth rate. It is apparent that there is strong similarity with the experimental results. In particular, the location of growth mode transition and the differing slopes of the second phase growth. However, despite using lateral mass limited growth which yields the sharpest possible transition achievable by our model we observe deviation in the first growth phase not present 

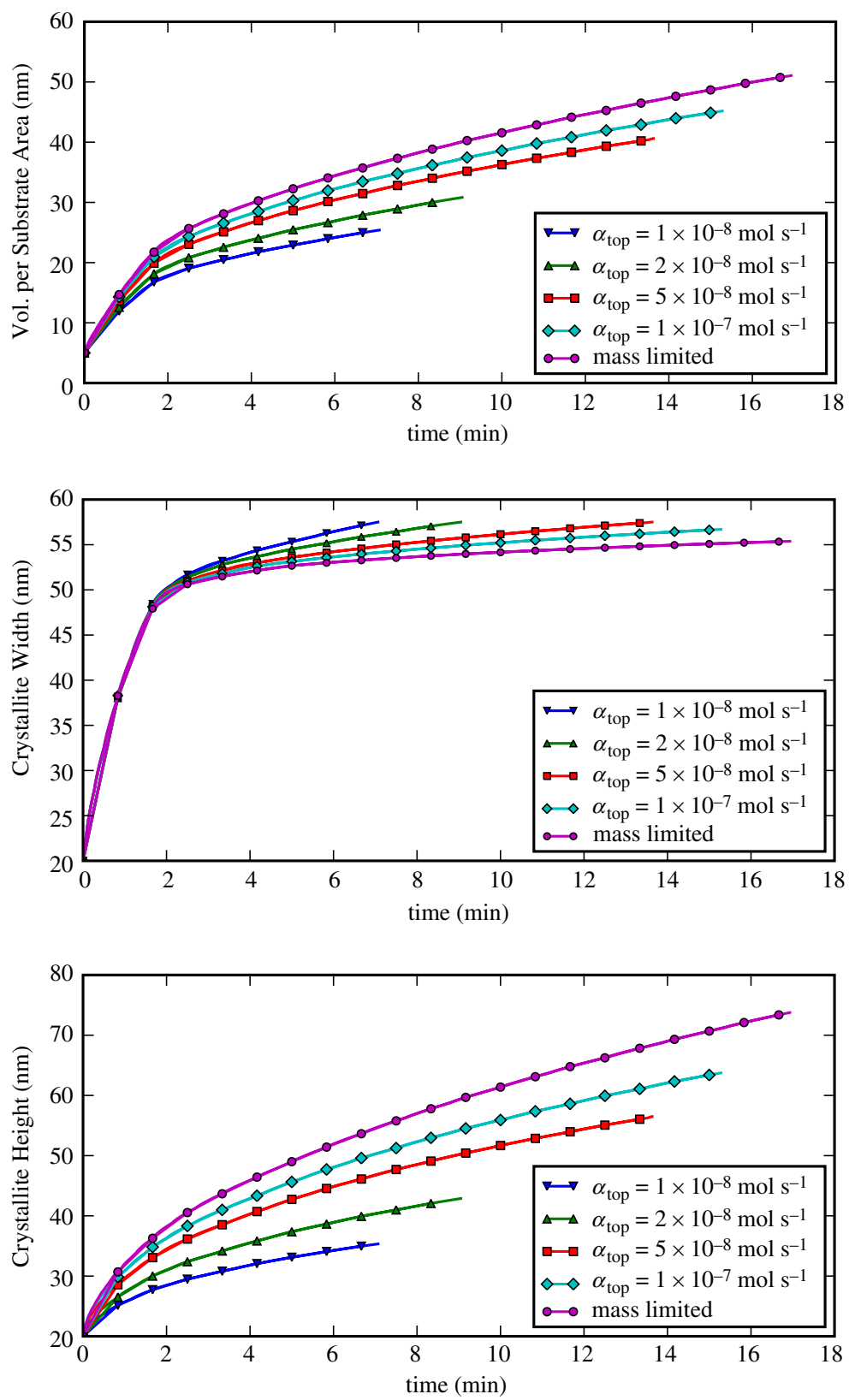

FIGURE 5. Simulated growth profiles of $\mathrm{ZnO}$ under mass transport limited lateral growth conditions for a variety of $\alpha_{\text {top }}$ values. Shown are simulation results for (top) crystallite growth, (middle) crystal width and (bottom) height decomposed simulation results for crystallite growth. The traces show that greater $\alpha_{\text {top }}$ results in slowed lateral growth. 


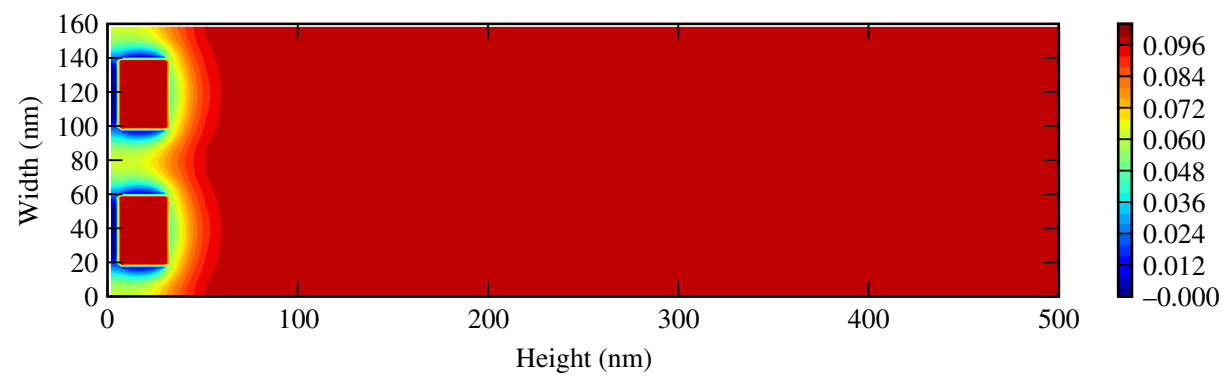

(a) $\mathrm{Zn}^{2+}$ concentration field at time $t=100 \mathrm{~s}$.

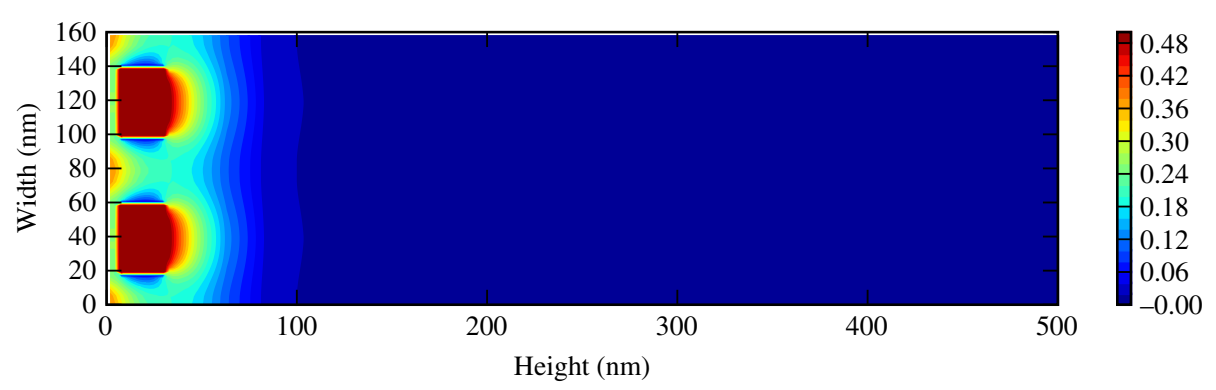

(b) $\mathrm{OH}^{-}$concentration field at time $t=100 \mathrm{~s}$.

FIGURE 6. Concentration fields for $\mathrm{Zn}^{2+}$ and $\mathrm{OH}^{-}$shortly before growth mode transition at time $t=100$ $\mathrm{s}$ for $\alpha_{\mathrm{top}}=1 \times 10^{-7} \mathrm{~mol} \mathrm{~s}^{-1}$. The scale bars both have units of $\mathrm{mol} \mathrm{L}^{-1}$.

in the experimental measurements, which exhibit a very clear transition between the two growth phases. This may be due to the two-dimensional nature of our simulation which does not weight the contribution of the side growth as strongly as it would be in the experiments. Figure 6 shows the concentrations of $\mathrm{Zn}^{2+}$ and $\mathrm{OH}^{-}$shortly before the switch in growth mode. We observe that the lateral growth has virtually ceased due to the consumption of all $\mathrm{Zn}^{2+}$ and $\mathrm{OH}^{-}$and the inter-rod channel is too small for significant $\mathrm{Zn}^{2+}$ or $\mathrm{O}_{2}$ replenishment. It seems likely that consumption of $\mathrm{Zn}^{2+}$ between the rods may also be the cause of the two-phase growth seen experimentally [7].

\section{Discussion and conclusion}

We have shown that the model developed here reproduces the experimentally observed two-mode growth behaviour and the time at which the transition between modes occurs. Our simulation using the diffusion limited oxygen reduction reaction condition on both the electrode and crystallite faces is close enough to the experimental result for us to be confident that the numerical model is capable of replicating experiment and predicting new behaviour under different conditions. Generally good quantitative agreement is found between the model predictions and recent 
experimental results, suggesting that the two-mode growth seen in the experiments is caused by a transition from three-dimensional to one-dimensional growth as the $\mathrm{Zn}^{2+}$ is consumed between the rods.

\section{Acknowledgements}

Funding was provided in part by the New Zealand Foundation for Research, Science and Technology under contract C08X0409. Unfortunately Stephen White passed away in 2006 before the bulk of this work was complete. Nonetheless, Steve played a vital role in the formulation and methodology used in this paper. Without his contribution this work would not have been possible.

\section{References}

[1] M. S. Arnold, P. Avouris, Z. W. Pan and Z. L. Wang, "Field-effect transistors based on single semiconducting oxide nanobelts", J. Phys. Chem. B 107 (2003) 659-663.

[2] G. Aylward and T. Findlay, SI chemical data, 3rd edn (Wiley, Brisbane, 1994).

[3] B. Canava and D. Lincot, "Nucleation effects on structural and optical properties of electrodeposited zinc oxide on tin oxide", J. Appl. Electrochem. 30 (2000) 711-716.

[4] D. Gal, G. Hodes, D. Lincot and H.-W. Schock, "Electrochemical deposition of zinc oxide films from non-aqueous solution: a new buffer/window process for thin film solar cells", Thin Solid Films 361 (2000) 79-83.

[5] A. Goux, T. Pauporte and D. Lincot, "Temperature effects on ZnO electrodeposition", Electrochim. Acta 50 (2005) 3168-3172.

[6] B. Illy, B. A. Shollock, J. L. MacManus-Driscoll and M. P. Ryan, "Electrochemical growth of ZnO nanoplates", Nanotechnology 16 (2005) 320-324.

[7] B. Ingham, B. N. Illy, J. R. Mackay, S. P. White, S. C. Hendy and M. P. Ryan, "In situ synchrotron $\mathrm{X}$-ray absorption experiments and modelling of the growth rates of electrochemically deposited ZnO nanostructures", Mater. Res. Soc. Symp. Proc. 1017 (2007) DD12-DD16.

[8] M. Izaki and T. Omi, "Transparent zinc oxide films prepared by electrochemical reaction", Appl. Phys. Lett. 68 (1996) 2439-2440.

[9] M. Kadota and T. Miura, "Shear bulk wave transducer made of (1120)-plane epitaxial $\mathrm{ZnO}$ film on r-sapphire", Japan J. Appl. Phys. 41 (2002) 3281-3284.

[10] M. Law, L. E. Greene, J. C. Johnson, R. Saykally and P. D. Yang, "Nanowire dye-sensitized solar cell", Nature Mater. 4 (2005) 455-459.

[11] J. Lee and Y. Tak, "Electrodeposition of $\mathrm{ZnO}$ on ITO electrode by potential modulation method", Electrochem. Solid State Lett. 4 (2001) C63-C65.

[12] D. R. Lide (ed), CRC handbook of chemistry and physics, 86th edn (CRC Press, Boca Raton, FL, 2004).

[13] R. V. A. Liu, E. Bohannan, T. Sorensen and J. Switzer, "Epitaxial electrodeposition of ZnO nanopillars on single-crystal gold", Chem. Mater. 13 (2001) 508-512.

[14] B. O'Regan, V. Sklover and M. Gratzel, "Electrochemical deposition of smooth and homogeneously mesoporous $\mathrm{ZnO}$ films from propylene carbonate electrolytes", J. Electrochem. Soc. 148 (2001) C498-505.

[15] R. K. Pandy, S. N. Sahu and S. Chandra, Handbook of semiconductor electrodeposition, 1st edn (Marcel Dekker Inc., New York, 1996).

[16] S. Peulon and D. Lincot, "Cathodic electrodeposition from aqueous solution of dense or openstructured zinc oxide films", Adv. Mater. 8 (1996) 166-170.

[17] S. P. White, G. J. Weir and N. J. Laycock, "Calculating the chemical concentrations during the initiation of crevice corrosion", Corros. Sci. 42 (2000) 605-629. 
[18] M. H. Wong, A. Berenov, X. Qi, M. J. Kappers, Z. H. Barber, B. Illy, Z. Lockman, M. P. Ryan and J. L. MacManus-Driscoll, "Electrochemical growth of $\mathrm{ZnO}$ nano-rods on polycrystalline $\mathrm{Zn}$ foil", Nanotechnology 14 (2003) 968-973.

[19] E. Yeager, J. O'M. Bockris, B. E. Conway and S. Sarangapani (eds), Comprehensive treatise of electrochemistry, 1st edn, Volume 6 (Plenum Press, New York, 1983). 